\title{
Correction to: Experimental investigation of Görtler vortices in hypersonic ramp flows
}

\author{
Amit Roghelia $^{1}\left(\mathbb{D} \cdot\right.$ Herbert Olivier $^{1} \cdot$ Ivan $\operatorname{Egorov}^{2} \cdot$ Pavel Chuvakhov $^{2}$
}

Published online: 13 November 2017

(C) Springer-Verlag GmbH Germany 2017

Correction to: Exp Fluids (2017) 58:139

https://doi.org/10.1007/s00348-017-2422-y

Figure 6a of the original article is published incorrectly. Specifically, the notation for radius of curvature $\mathbf{R}$ is missing. The correct figure is shown below.

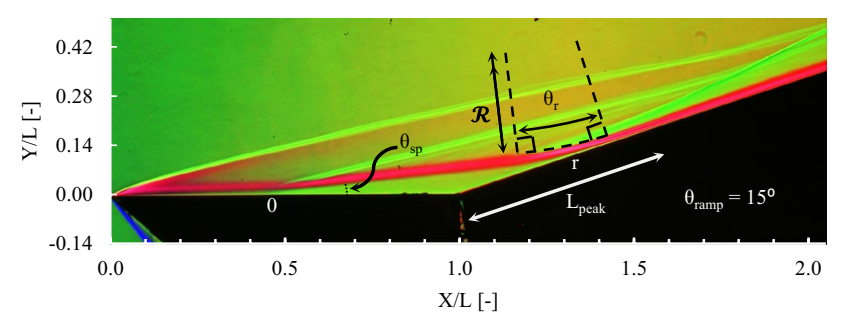

The original article can be found online at https://doi.org/10.1007/s00348-017-2422-y.

Amit Roghelia

roghelia@swl.rwth-aachen.de

http://www.swl.rwth-aachen.de

1 Shock Wave Laboratory, RWTH Aachen University, Templergraben 55, 52062 Aachen, Germany

2 Central Aerohydrodynamic Institute (TsAGI), 1 Zukovskogo Str, Zhukovsky, Moscow Region 140180, Russia 